\title{
Velocity and turbulence measurements of oil-water flow in horizontal and slightly inclined pipes using PIV
}

\author{
W. A. S. Kumara ${ }^{1}$, B. M. Halvorsen ${ }^{1,2}$ \& M. C. Melaaen ${ }^{1,2}$ \\ ${ }^{I}$ Telemark University College, Norway \\ ${ }^{2}$ Telemark $R \&$ D Centre (Tel-Tek), Norway
}

\begin{abstract}
Oil-water flows in horizontal and slightly inclined pipes are investigated using Particle Image Velocimetry (PIV). PIV offers a powerful non-invasive tool to study such flow fields. The experiments are conducted in a $15 \mathrm{~m}$ long, $56 \mathrm{~mm}$ diameter, inclinable steel pipe using Exxsol D60 oil (viscosity $1.64 \mathrm{mPa} \mathrm{s}$, density $790 \mathrm{~kg} / \mathrm{m}^{3}$ ) and water (viscosity $1.0 \mathrm{mPa} \mathrm{s}$, density $996 \mathrm{~kg} / \mathrm{m}^{3}$ ) as test fluids. The test pipe inclination is changed in the range from $5^{\circ}$ upward to $5^{\circ}$ downward. The experiments are performed at mixture velocity $0.25 \mathrm{~m} / \mathrm{s}$ and inlet water volume fraction 0.25 . The instantaneous local velocities are measured using PIV, and based on the instantaneous local velocities mean velocities and turbulence profiles (U-rms, V-rms and Reynolds stresses) are calculated. The time averaged cross sectional distributions of oil and water phases are measured with a traversable gamma densitometer. The flow regimes are determined based on visual observations. The measured flow regimes, water hold-up, slip ratio and velocity and turbulence profiles show a strong dependency with pipe inclination.

Keywords: oil-water flow, horizontal flow, inclined flow, particle image velocimetry, gamma densitometry, hold-up, slip ratio, turbulence measurements.
\end{abstract}

\section{Introduction}

The flow of two immiscible liquids is often encountered in many industrial applications, especially in the petroleum industry. However, despite their abundance, liquid-liquid flow systems have not drawn so much attention as gasliquid flows. Only recently, they are attracting more and more interest due to 
continuous improvements required by technological applications in offshore oil industry. The current trend in offshore oil production is characterized by deepwaters, smaller oil fields with thin oil layers, increased water production and development of horizontal and branched wells to easily penetrate into the large areas of the reservoir. Offshore deep-water production would involve transportation of oil-water mixtures from the wellhead to a central gathering station or a platform as shown schematically in fig. 1. The terrain is not flat and flows through inclined pipes are often encountered. Oil-water mixtures may sometimes be transported through hilly terrain as well. The pipe inclination may lead to more mixing of the oil-water phases, and flow patterns, phase distributions and pressure drop will be highly affected. The knowledge about the hydrodynamic properties associated with these flows is extremely important to ensure safe design and efficient operation of offshore transportation pipelines. Nevertheless, limited number of studies on oil-water flow in inclined pipes has been performed in the past [1-4]. The existing literature covers mainly the flow patterns, pressure drop and hold-up measurements of oil-water flow in inclined pipes. The data on mean velocities, velocity fluctuations, Reynolds stresses and other turbulence properties have not been measured with sufficient details and accuracy. The present paper reports the mean axial velocity and turbulence measurements of oil-water flow in pipes at different pipe inclinations $\left(-5^{\circ},-1^{\circ}\right.$, $0^{\circ},+1^{\circ}$ and $\left.+5^{\circ}\right)$ using Particle Image Velocimetry (PIV). The time averaged cross-sectional distribution of oil and water is measured using a gamma densitometer. The flow regimes are determined by visual observations.

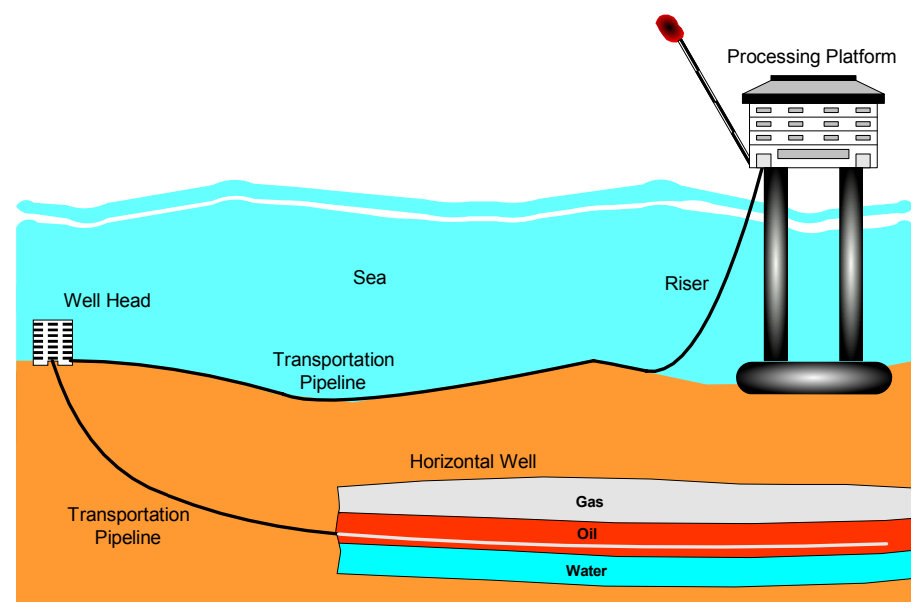

Figure 1: Schematic of a subsea processing facility.

\section{Experimental set-up}

The experiments were carried out in the multiphase flow facility at Telemark University College, Porsgrunn, Norway. 


\subsection{Multiphase flow loop}

A simplified flow sheet of the experimental rig is shown in fig. 2.

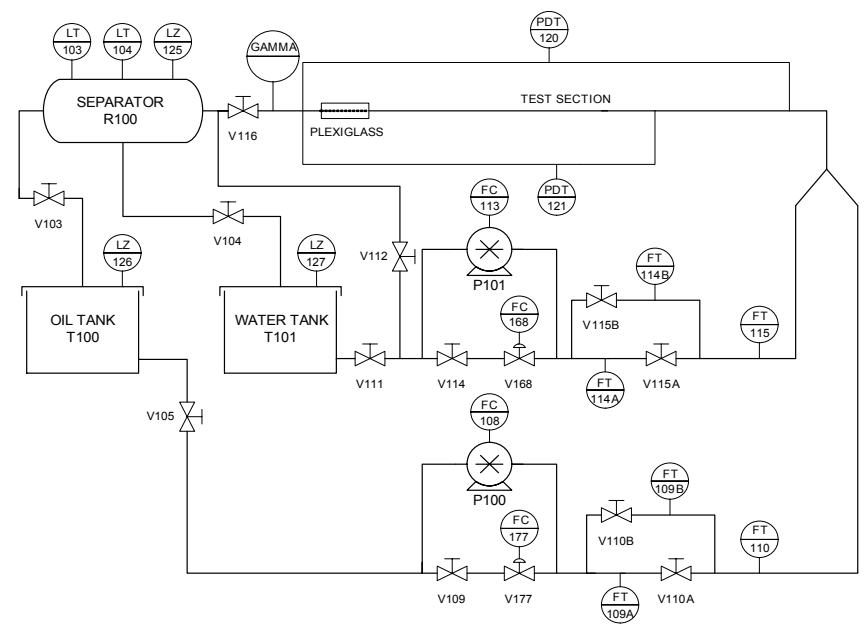

Figure 2: $\quad$ Simplified flow sheet for the test rig.

The experiments were performed using water (density $996 \mathrm{~kg} / \mathrm{m}^{3}$, viscosity $1 \mathrm{mPa} \mathrm{s}$ ) and Exxsol D60 oil (density $790 \mathrm{~kg} / \mathrm{m}^{3}$, viscosity $1.64 \mathrm{mPa}$ s) as test fluids. Oil and water are stored in separate tanks (T100 and T101 for oil and water, respectively) and circulated using volumetric pumps P100 and P101. The mass flow, density and temperature are measured for each phase before entering the test section using Coriolis flow meters (FT109B, FT110, FT114B and FT115). A controller based on LabView $^{\circledR}$ allowed to set the input oil and water flow rates and to select the appropriate pumps and flow meters. The test section is a $15 \mathrm{~m}$ long steel pipe with inner diameter equal to $56 \mathrm{~mm}$. The test pipe inclination is changed in the range from $5^{\circ}$ upward to $5^{\circ}$ downward. Towards the end of the test section, there is a short transparent section for visual observations and PIV measurements.

\subsection{Particle Image Velocimetry (PIV)}

The fundamental methodology employed in the PIV system is relatively straightforward and actually rather simple in principle. The PIV measurements include illuminating a cross section of the seeded flow field, typically by pulsing light sheet, recording multiple images of the seeding particles in the flow using a camera located perpendicular to the light sheet, and analyzing the images for displacement information. The velocity and turbulence distributions are estimated based on particle displacements. The PIV system used for this study consists of a pulsed laser, CCD video camera, synchronizing system and a personal computer all linked to each other. The illumination beam is produced by dual cavity Nd:YAG laser with peak emission of $532 \mathrm{~nm}$ waves with the output 
energy of $50 \mathrm{~mJ} /$ pulse. The laser beam is directed through an optical system to produce a thin planer sheet of high intensity laser light. This sheet of laser light is aligned to illuminate the vertical plane across the pipe center. The oil and water phases are seeded for PIV measurements. In general, these particles should be small enough to be good flow tracers and large enough to scatter sufficient light for imaging. Polyamide seeding particles (PSP) having mean particle diameter of $20 \mu \mathrm{m}$ are used in both oil and water phases. The flow images are recorded with a X-Stream ${ }^{\mathrm{TM}} \mathrm{XS}-3$ cross correlation CCD camera of $1260 \times 1024$ pixel resolution. The camera is focussed normal to the illuminated flow field. A Dantech FlowMap processor is used to synchronize the laser and camera and to process the resulting image pairs. The PIV system allows the acquisition of flow images with the sample frequency of $50 \mathrm{~Hz}$. The first and higher order turbulence statistics are estimated based on 4084 flow images. The flow images as shown in fig. 3(a) are divided into rectangular regions called interrogation areas. In the present experiments, the interrogation area is $32 \times 32$ pixels, which yields to a spatial resolution of $1.92 \times 1.92 \mathrm{~mm}$. For each of these interrogation areas the images from the first and second pulse of the light sheet are crosscorrelated to produce average particle displacement vectors. The instantaneous velocity vectors are estimated based on average displacement vectors and time delay between laser pulses. From a series of instantaneous velocity measurements, the mean velocity and turbulence properties can be estimated. The number of tracer particles in the flow is very important in obtaining a good signal peak in the cross correlations. As a rule of thumb, 10 to 25 particle images should be seen in each interrogation area. Fig. 3(b) shows distribution of tracer particles in four interrogation areas.

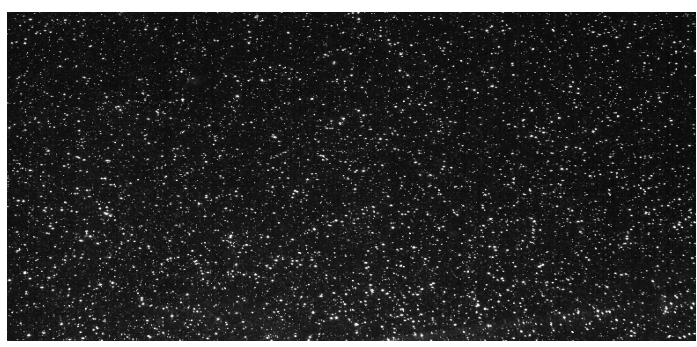

(a)

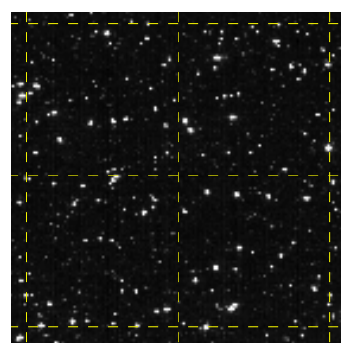

(b)

Figure 3: PIV flow images: (a) Particle image, (b) Tracer particles in four interrogation areas.

\subsection{Gamma densitometry}

The gamma densitometry offers non-intrusive measurements of local phase distributions in oil-water flow. It exploits the fact that electromagnetic radiation is attenuated as it passes through matter owing to the interaction of its photons with the matter. The gamma densitometer is equipped with a $45 \mathrm{mCi}$ Americium-241 source and a detector [sodium iodide (NaI) scintillation crystal 
doped with thallium]. The source and the detector are located diametrically opposite to each other on a pipe section with collimator structure used to ensure the production of a narrow gamma beam. The degree of attenuation experienced by a narrow beam of gamma radiation is a function of the gamma beam photons' energy and the density of the absorbing matter. In two-phase flow, this can be calibrated to measure the local water volume fraction in the volume covered by the gamma beam. By traversing the gamma densitometer, the cross-sectional distribution of oil and water phases can be measured. In the experiments, the vertical interface position is measured by traversing horizontal gamma beams.

\subsection{Investigated flow conditions}

All the experiments are performed at mixture velocity $0.25 \mathrm{~m} / \mathrm{s}$ and inlet water cut 0.25 . The mixture velocity for oil-water flow is defined as:

$$
U_{m}=\frac{Q_{o}+Q_{w}}{A}
$$

where $Q_{o}$ and $Q_{w}$ are the inlet volumetric flow rates of oil and water, respectively and $A$ is the pipe cross-sectional area. The inlet water cut $\left(\lambda_{w}\right)$ is defined as:

$$
\lambda_{w}=\frac{Q_{w}}{Q_{o}+Q_{w}}
$$

The experiments are performed at five different pipe inclinations $\left(-5^{\circ},-1^{\circ}, 0^{\circ}\right.$, $+1^{\circ}$ and $\left.+5^{\circ}\right)$.

\section{Results and discussion}

The effect of pipe inclinations on the flow patterns, hold-up, slip ratio, velocity and turbulence in oil-water flow is investigated in this section.

\subsection{Water hold-up and slip ratio measurements}

The water hold-up $\left(\eta_{w}\right)$ for oil-water flow is defined as:

$$
\eta_{w}=\frac{A_{w}}{A}
$$

where $A_{w}$ is the cross sectional area occupied by water phase. The vertical distance from the bottom of the pipe to the point, where the local water volume fraction is equal to 0.50 is considered as the interface height and the interface is treated as a flat surface in order to estimate the flow areas for different phases. It is possible to measure the liquid fractions as function of time at a given cross section of the pipe: the so-called in-situ water hold-up. In oil-water flows, the insitu hold-up will be time-dependent due to, for example, wave motion and mixing at the interface. An averaged hold-up value can be obtained by taking some average of the time dependent signal. The water hold-up values reported in this paper is estimated using time averaged local water volume fraction measurements from gamma densitometry. 
The ratio between the averaged in-situ velocities of the two phases is often given as slip ratio, $S$, and it can be calculated as follows:

$$
S=\frac{U_{o}}{U_{w}}=\frac{A_{w}}{A_{o}} \frac{U_{s o}}{U_{s w}}
$$

where $\mathrm{A}_{\mathrm{o}}$ is the flow area occupied by the oil phase. $U_{s o}$ and $U_{s w}$ are superficial velocities for oil and water phases, respectively. The superficial velocities for oil and water phases are defined as follows:

$$
U_{s o}=\frac{Q_{o}}{A} \text { and } U_{s w}=\frac{Q_{w}}{A}
$$

The slip ratio is dependent on physical properties combined with flow rates, flow pattern and pipe geometry. $S>1$ means that the oil travels faster than water in the pipe while $S<1$ indicates that water is the faster phase.

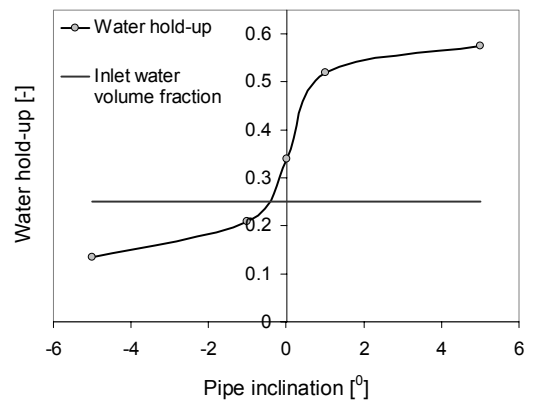

(a)

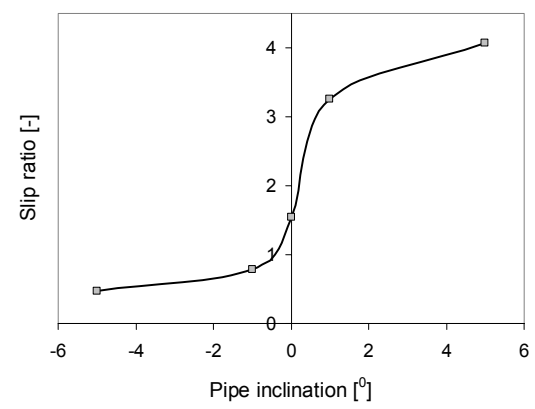

(b)

Figure 4: The water hold-up and slip-ratio as a function of the pipe inclination: (a) Water hold-up, (b) Slip ratio.

Fig. 4 (a) shows the variation of water hold-up of oil-water flow as a function of pipe inclination at mixture velocity $0.25 \mathrm{~m} / \mathrm{s}$ and inlet water cut 0.25 . The constant inlet water volume fraction used in all the experiments is also indicated. It is important to note that the hold-up which is measured, is in almost all instances significantly different from the input water volume fraction. In upward flow there is an expected trend of in-situ accumulation of the denser phase, in this case water. Hence, the measured water hold-up reaches higher values compared to horizontal flow as can be seen in the fig. 4(a). The measured holdup value for horizontal $\left(0^{\circ}\right)$ flow is 0.34 and it increases up to 0.52 and 0.58 for upward flow at $+1^{\circ}$ and $+5^{\circ}$. The water hold-up increase from $0^{\circ}$ to $+1^{\circ}$ appears greater than from $+1^{\circ}$ to $+5^{\circ}$. In addition, it is very dependent on the pipe inclination when the pipe is nearly horizontal. The physical reason for this is as follows. There is a shearing force at the interface separating the oil and the water due to the velocity difference between these phases. At inlet water cut 0.25 , the oil moves faster than the water so that the water is dragged along by the oil 
phase. When the pipe has an upward inclination, gravity will act to pull the more dense water phase downwards. Thus, the total force dragging the water up the inclined pipe is now reduced due to the action of the gravitational force. As a result, the water moves slower. With the constant volume flow of fluids and conservation of mass, a slower water velocity implies that the cross section of the pipe occupied by the water phase must increase. In other words, the water hold-up increases. When the pipe is inclined downwards, the opposite will happen: gravity works in the same direction as the shearing force and water holdup is low compared to the horizontal flow. The measured water hold-up values are 0.21 and 0.14 for pipe inclination angles $-1^{\circ}$ and $-5^{\circ}$, respectively. The deviation of measured water hold-up values from input water volume fraction is higher for upward flow compared to the downward flow as shown in fig. 4(a).

The slip ratio of oil-water flow in horizontal and slightly inclined pipes is presented in fig. 4(b) at mixture velocity 0.25 and inlet water cut 0.25 . It shows some similarities with the hold-up distribution presented earlier, giving an indication of the strong link between the water hold-up and the slip ratio of oilwater flow in pipes. The measured slip ratio for horizontal flow is 1.54 . In this case, oil flows faster than water phase due to difference in wetted perimeter. The slip ratio varies widely with pipe inclination for near horizontal flows. The measured slip ratios are 3.25 and 4.08 for pipe inclinations $+1^{\circ}$ and $+5^{\circ}$, respectively. At low inlet water fraction $\left(\lambda_{\mathrm{w}}=0.25\right)$, the oil, as the less dense phase, travels at a considerably higher velocity than the water, so that $S$ is always above one for upwardly inclined flows. Previous studies indicate that at low upward inclination generally results in higher water hold-up than in horizontal flow giving higher slip ratios [5-7]. The relevant systems are described by Scott [5] at $+15^{\circ}$ and $+30^{\circ}$, Lum et al [6] at $+5^{\circ}$ and Abduvayt et al [7] at $+0.5^{\circ}$ and $+3^{\circ}$. The reported $S$ values are in general above one, for upwardly inclined oilwater flows and show a good agreement with present observations. In addition, the measurements show that the slip ratio increases largely when the pipe is inclined from $0^{\circ}$ to $+1^{\circ}$ compared to from $+1^{\circ}$ to $+5^{\circ}$. This could be due to the increased mixing at $+5^{\circ}$, higher water hold-up is still favoured but is tempered slightly by the increased level of mixing and interfacial waves.

The slip ratios of downwardly inclined pipes show fewer deviations from horizontal flow compared to the upwardly inclined pipes. The measured slip ratios for pipe inclinations $-1^{\circ}$ and $-5^{\circ}$ are 0.79 and 0.47 . In downwardly inclined oil-water flows at these inclination angles, the gravity would favour faster water flow and the slip ratio is below one. The available data on slip ratio measurements in downward oil-water flow are by Cox [8] at $-15^{\circ}$ and $-30^{\circ}$ and Abduvayt et al [7] at $-0.5^{\circ}$ and $-3^{\circ}$. Abduvayt et al [7] showed that the slip ratio is always less or equal to one for downwardly inclined oil-water flows. Nevertheless, Cox [8] reported generally low slip ratios, although not always below one. The present slip ratio profile shows that the slip ratio can be above one for downwardly inclined oil-water flow at very small pipe inclinations. Hence, the present measurements show a good accordance with data presented by Cox [8]. 


\subsection{Local water volume fraction, velocity and turbulence measurements}

The PIV measurements are performed on two-phase oil-water flow at mixture velocity $0.25 \mathrm{~m} / \mathrm{s}$ and inlet water cut 0.25 for five different pipe inclinations. The measurements of mean axial velocity, root mean squared velocities and Reynolds shear stresses are presented. In every plot, the local water volume fraction data from gamma densitometry is included to indicate the position of the interface. The still flow images are used as the background of the graphical representation of measured data for better visualization of the flow.

\subsubsection{Horizontal flow}

Fig. 5 presents local water volume fraction, mean axial velocity and turbulence profiles for oil-water flow in horizontal pipe. The direction of the flow is from left to right with the oil phase in the upper part of the pipe. The flow regime in this case is smooth stratified flow without dispersion. The flow is gravity dominated and the phases are segregated having a smooth interface.

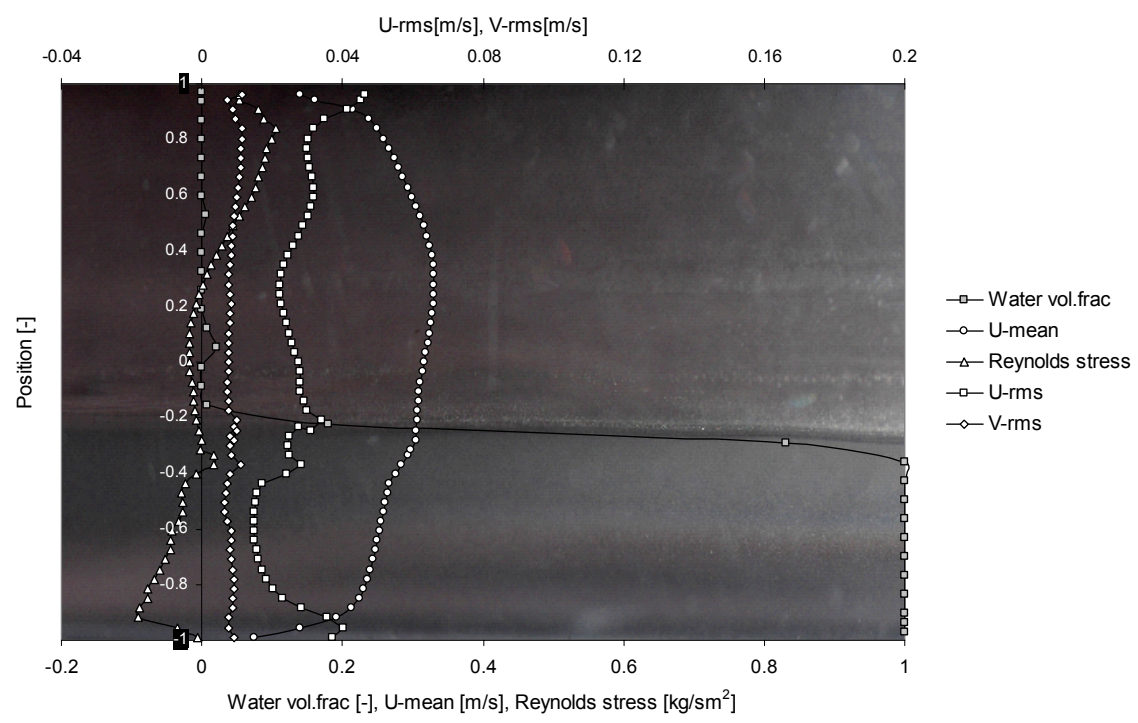

Figure 5: Mean axial velocity and turbulence measurements for horizontal flow.

The local water volume fraction is expected to be zero in the oil phase and one in the water phase. At the interface it will be somewhere in between. Good agreement between gamma densitometry measurements and visual observations can be seen. The mean axial velocity profile shows that mean axial velocity is highest in the oil phase, as expected. In this case, the Reynolds numbers for oil and water phases are 5685 and 8344, respectively. The turbulence statistics of the flow field are calculated based on instantaneous velocity measurements. The root mean-squared velocity components of streamwise (U-rms) and wall normal 
(V-rms) directions are presented together with Reynolds stress $(\rho \overline{u v})$. Here, $\mathrm{V}$ is the mean velocity component in wall normal direction, $u$ and $v$ represent the fluctuating velocity components in streamwise and wall normal directions and $\rho$ is the fluid density. The root mean squared velocity components represent time averaged values of fluctuating velocities. The largest values of U-rms, V-rms and Reynolds stress are observed in the regions where the mean axial velocity gradient is largest highlighting the intimate connection between turbulence production and sheared mean flow. The streamwise intensity, U-rms, is produced by mean shear and two peaks are observed very close to the wall in oil and water phases. The wall normal stress component, V-rms, is fed by redistribution of intensity from U-rms, hence fall below U-rms. The difference between U-rms and V-rms implies an anisotropic structure of the turbulence. The maximum mean axial velocity is observed at normalized radial position 0.33 in the oil phase. At this point, the mean axial velocity gradient is zero and hence no turbulence is produced. Nevertheless, the values of U-rms and V-rms do not decrease very much because vigorous eddy mixing transports turbulent fluid from nearby regions of high turbulence production towards this region. A slight increase in U-rms is observed close to the interface. The impenetrability of the pipe wall suppresses the normal component of the turbulence. Therefore the maximum values of U-rms are observed very close to the wall whereas the maximum values of Reynolds stress are located at a certain distance away from the pipe wall. The Reynolds stress is proportional to the shear rate and it can be expressed as:

$$
\rho \overline{u v}=-\mu_{t} \frac{\partial U}{\partial y}
$$

where $\mu_{t}$ is the turbulent eddy viscosity. The expression is often known as Boussinesq eddy viscosity hypothesis. As shown in fig. 5, the mean velocity gradient is positive in the water phase and Reynolds stress is negative. This follows the above argument based on Boussinesq eddy viscosity hypothesis. The Reynolds stress is zero at the point where the maximum mean axial velocity is observed. This is due to the change of the sign of the mean velocity gradient. The Reynolds stress has a special behaviour close to the oil-water interface. It decreases towards zero close to the interface. The oil-water interface acts as a moving wall and the stable density stratification close to the interface suppresses turbulence fluctuations normal to the interface. This may be the reason for the observed smaller values of Reynolds stress close to the interface.

\subsubsection{Upward flow}

The effect of pipe inclination on flow pattern, local water volume fraction, mean axial velocity and turbulence profiles of upwardly inclined oil-water flows are presented in fig. 6. The measurements are performed at two different pipe inclinations, $+1^{\circ}$ and $+5^{\circ}$. The introduction of a small inclination in the pipeline, as well as the size of the inclination, has a significant impact on oil-water flow in pipes. The observed flow patterns at $+1^{\circ}$ and $+5^{\circ}$ are very different from the smooth stratified flow observed in the horizontal pipe for the same flow 
conditions. In deviated flows, the gravitational force has components both normal and parallel to the pipe axis. The normal component promotes segregation of the phases as in the horizontal flow, while the parallel one can act either in the direction of the flow (downward flow) or in the opposite direction (upward flow). Therefore, a declination in the pipe causes higher in-situ water velocity than in the corresponding horizontal case; conversely, the in-situ water velocity in an inclined pipe is lower than in a horizontal pipe. As shown in fig. 6(a) stratified flow with some mixing at the interface is observed at the pipe inclination $+1^{\circ}$. The parallel gravity component introduces significantly higher slip between oil and water phases at $+1^{\circ}$ compared to the horizontal flow as shown in fig. 4(b). The increased relative movement between the phases results in the development of vortex motion at the boundary of the two liquids. Mutual penetrations of vortices take place in each of the phases. This interface disturbances lead to the formation of liquid drops. The dynamic and buoyant forces are acting simultaneously on the drops. The former, which tend to spread the drops throughout the pipe section are not large enough to overcome the settling tendency of the counteracting gravity force and both kinds of drops remain close to the interface. Some interfacial waves are also observed. As shown in fig. 6(b) for inclination $+5^{\circ}$ the smooth interface of horizontal flow is completely replaced by large amplitude waves together with some mixing at the interface. The oil-water interface is noticeably wavier and more irregular at $+5^{\circ}$ than at $+1^{\circ}$.

In comparing the current observations with those of previous investigators there is a good consensus on the flow behaviour at this range of flow conditions. At low mixture velocities, where the transition from stratified to dual continuous flow occurs, a general agreement exists that in upward flow dispersion appears at lower velocities than in the horizontal case [5, 9 and 10]. The experimental data of Oddie et al [9] do indicate the enhancement of mixing with increased pipe inclination. Lum et al [2] have investigated oil-water flow patterns at mixture velocity $0.7 \mathrm{~m} / \mathrm{s}$ and inlet water fraction 0.32 for upwardly inclined pipes. They have reported stratified flow with smooth interface for horizontal flow whereas stratified wavy flow pattern has been observed in upwardly inclined pipes for similar conditions. The degree of waviness correspondingly increased with pipe inclination so that the flow at $+10^{\circ}$ is wavier than at $+5^{\circ}$. Therefore, the present observations closely adhere to the data presented by previous investigators on flow patterns of oil-water flow in upwardly inclined pipes.

Fig. 6(a) shows local water volume fraction, mean axial velocity and turbulence measurements at pipe inclination $+1^{\circ}$. The local water volume fraction measurements agree well with the visual observations. The oil layer at the top moves faster than the water layer and there is a sharp transition in velocity across the interface. The observed higher slip ratio $(S=3.25)$ causes an increase in the level of mixing at the oil-water interface. The maximum mean velocity is observed again in the oil phase and it is about $30 \%$ higher than the corresponding maximum velocity for horizontal flow. The estimated Reynolds numbers for oil and water phases are 10316 and 4208, respectively. The turbulence level in the oil phase has increased whereas it has decreased in the 


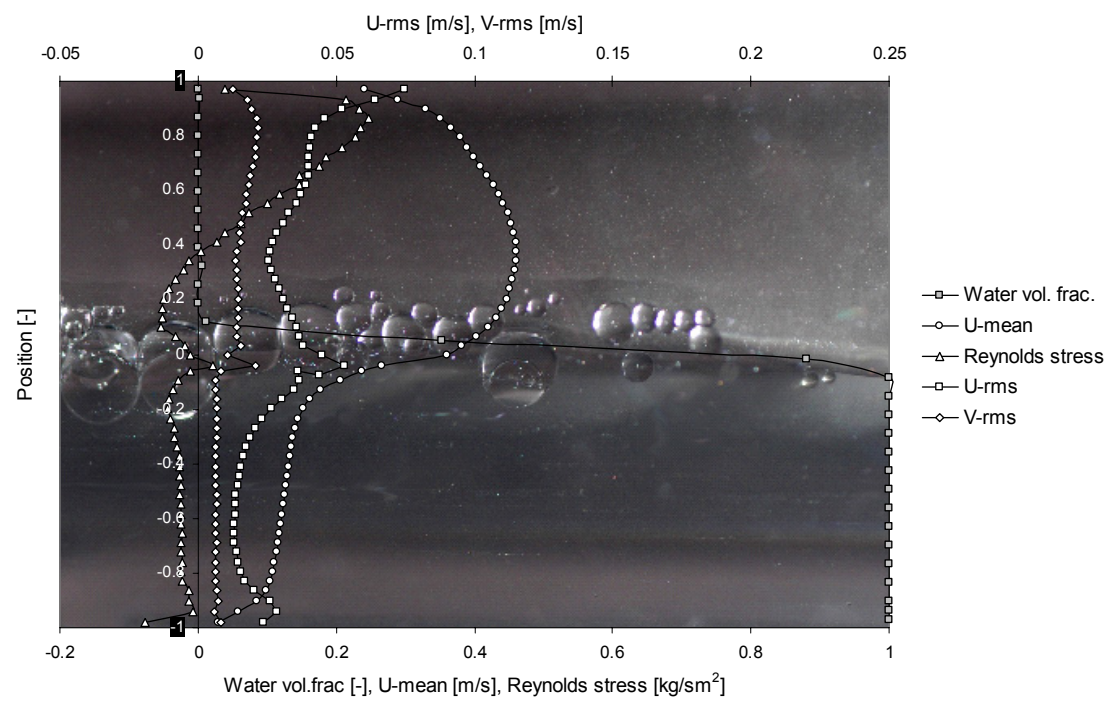

(a)

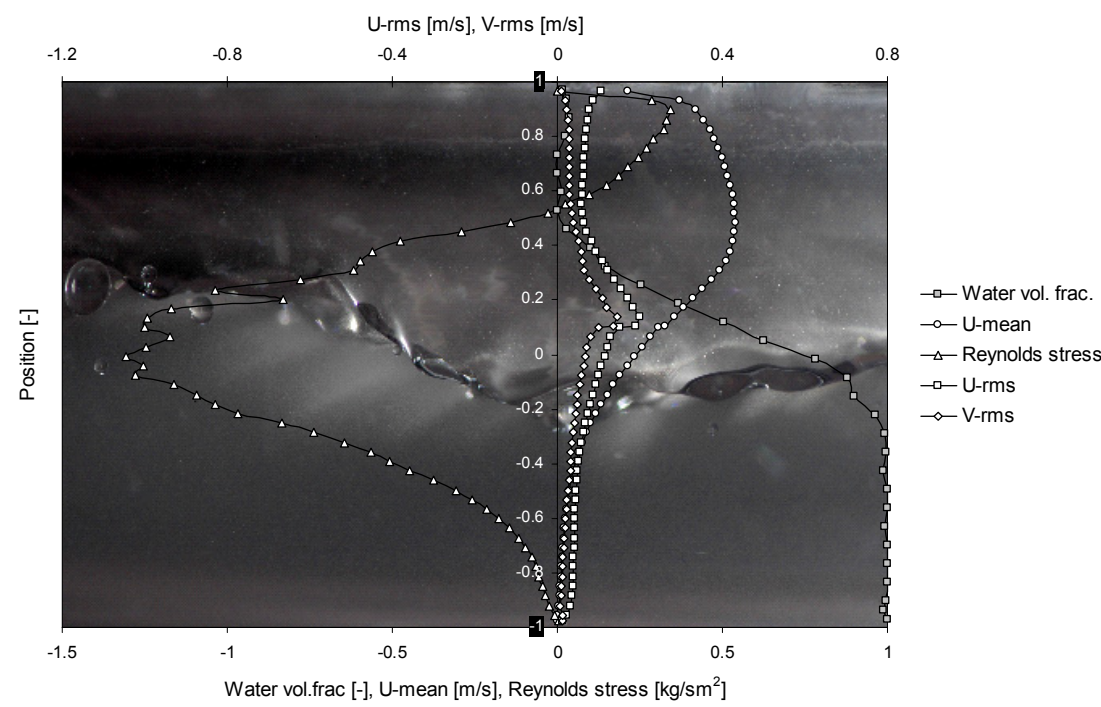

(b)

Figure 6: Mean axial velocity and turbulence measurements for upward flow: (a) $+1^{\circ}$, (b) $+5^{\circ}$.

water phase compared to the results for horizontal flow. The maximum U-rms value is located close to the wall in oil phase and it is about $30 \%$ of the mixture velocity and about $60 \%$ higher than the observed value for horizontal flow. The U-rms values show fewer variations over a wide range in the water phase due to 
relatively constant mean shear rate. The minimum value of U-rms in the oil phase is observed at the normalized radial position 0.34 where the mean axial velocity is largest. Some fluctuations of U-rms are observed at the interface. The measured V-rms values in the oil phase are slightly larger than the values in the water phase due to higher turbulence level. The V-rms profile is relatively flat in the water phase. The maximum Reynolds stress value is located close to the pipe wall in the oil phase and it is about $140 \%$ of the corresponding maximum vale observed for horizontal flow. The measured Reynolds stress profile changes its sign at the normalized radial position 0.34 and smaller values are observed close to the interface due to stable density stratification.

The local water volume fraction, mean axial velocity and turbulence measurements are presented in fig 6(b) for pipe inclination $+5^{\circ}$. The measured interface region by gamma densitometry extends from normalized position -0.29 to 0.52 . The sharp interface observed in horizontal flow and at $+1^{\circ}$ is absent and the interface is associated with transient effects due to waves and mixing. The gamma densitometry produces time-averaged measurements and it cannot capture the transient effects of the flow. The image used in fig. 6(b) shows the instantaneous flow behavior. Therefore, time averaged interface position measurements deviates from the visual observations. At pipe inclination $+5^{\circ}$ the water phase travels much slower than the oil phase. Most of water moves concurrently with the oil as it flows up the pipe. However, very close to the bottom of the pipe, gravity and frictional effects overcomes the flowing momentum of the water, resulting in the fall back of the water phase at the base of the pipe. This is the reason for the observed negative axial velocities very close to the wall in the water phase. The slip ratio further increases up to 4.08. The maximum mean axial velocity is located in the oil phase at normalized radial position 0.48 and it is about $63 \%$ higher than the corresponding value observed for horizontal flow. The Reynolds numbers for oil and water phases are 10944 and 4080, respectively. The measured U-rms and V-rms values are higher in the oil phase compared to the water phase. The measured U-rms value closest to the pipe wall in the oil phase is approximately $40 \%$ of the mixture velocity. The Urms and V-rms values slightly increase towards the interface both in oil and water phases. The measured Reynolds stress profile changes its sign at the normalized radial position 0.48 due to zero mean axial velocity gradient. Surprisingly, large Reynolds stress values are observed around the wavy oilwater interface in contrast with the results for horizontal and upwardly inclined flow at $+1^{\circ}$. As shown in fig. 5 and 6(a) smaller Reynolds stress values are observed close to the interface because of the damping effect of wall normal component of the turbulence fluctuations due to stable density stratification. As inclination is increased, the effect of gravitational forces tended to act strongly upon the fluid parallel to the pipe, but against flow, thus increasing the rolling motion of the phases around the interface generating interfacial waves. The rolling motion of the fluid around the interface enhances the turbulence fluctuations close to the interface. Therefore, U-rms, V-rms and Reynolds stress values increase towards the oil-water interface. 


\subsubsection{Downward flow}

Fig. 7 shows the measurements for downwardly inclined pipes. The experiments are performed at two different pipe inclinations, $-1^{\circ}$ and $-5^{\circ}$. At pipe inclination, $1^{\circ}$ stratified flow with clear interface is observed as shown in fig. 7(a). No sign of dispersion at the interface was observed in contrast with the results at $+1^{\circ}$. At $5^{\circ}$, the mixing between the two phases is enhanced, and the flow regime can be identified as stratified flow with mixing at the interface. A clear thin water layer is observed at the bottom of the pipe. In contrast to the current work, stratified wavy flow pattern has been observed in downward flow by other investigators [7-9]. This could be due to the different ranges of velocities, pipe diameters and liquid properties used. The present measurements show an extensive level of increase in mixing between oil and water phases at $-5^{\circ}$ compared to $+5^{\circ}$ and it has bean observed previously by Lum et al [6].

Fig. 7(a) shows local water volume fraction, mean axial velocity and turbulence measurements at pipe inclination $-1^{\circ}$. A good consensus is observed between gamma measurements and visual observations. The PIV measurements of mean axial velocity profile show the maximum velocity in the water phase as expected. The maximum mean axial velocity is located at normalized radial position -0.58 and it is very close to the oil-water interface. The fast moving water phase drags the oil layer and mean axial oil velocity increases towards the interface. The estimated Reynolds numbers for oil and water phases are 5387 and 10199, respectively. The turbulence intensity has been increased in the water phase whereas it has been decreased in the oil phase compared to the horizontal flow. The highest U-rms value is now observed in the water phase and it is about $20 \%$ of the mixture velocity. The maximum values of $\mathrm{V}$-rms in oil and water phases are approximately equal. The highest magnitude of the Reynolds stress is located in the water phase and is about $70 \%$ higher than the corresponding value in the oil phase. The measured axial velocity profile is having smaller gradients around normalized radial position 0.10 . It may be the reason for the observed smaller Reynolds stress values in that region. In addition, the Reynolds stresses are damped around the interface due to stable gravity stratification.

Fig. 7(b) presents measurements at pipe inclination $-5^{\circ}$. A thick interface region is visually observed due to increased mixing at the interface and it is validated by gamma measurements. The measured local water volume fraction values close to the bottom pipe wall is one and it indicates the existence of the thin continuous water layer. The thin water layer moves considerably faster than the oil phase. The measured maximum mean axial velocity in the water phase is about $25 \%$ higher than the corresponding value at $-1^{\circ}$. The mean axial velocity in the oil phase increases towards the interface. The calculated oil and water phase Reynolds numbers are 5253 and 12056, respectively. The U-rms value measured closest to the wall in the water phase is about $26 \%$ of the mixture velocity. PIV gives smooth continuous turbulence measurements in the oil phase whereas the measurements in the thin oil layer and close to the interface show some fluctuations. The measured values of both U-rms and V-rms slightly increase towards the interface. Higher Reynolds stress values are observed close to the 


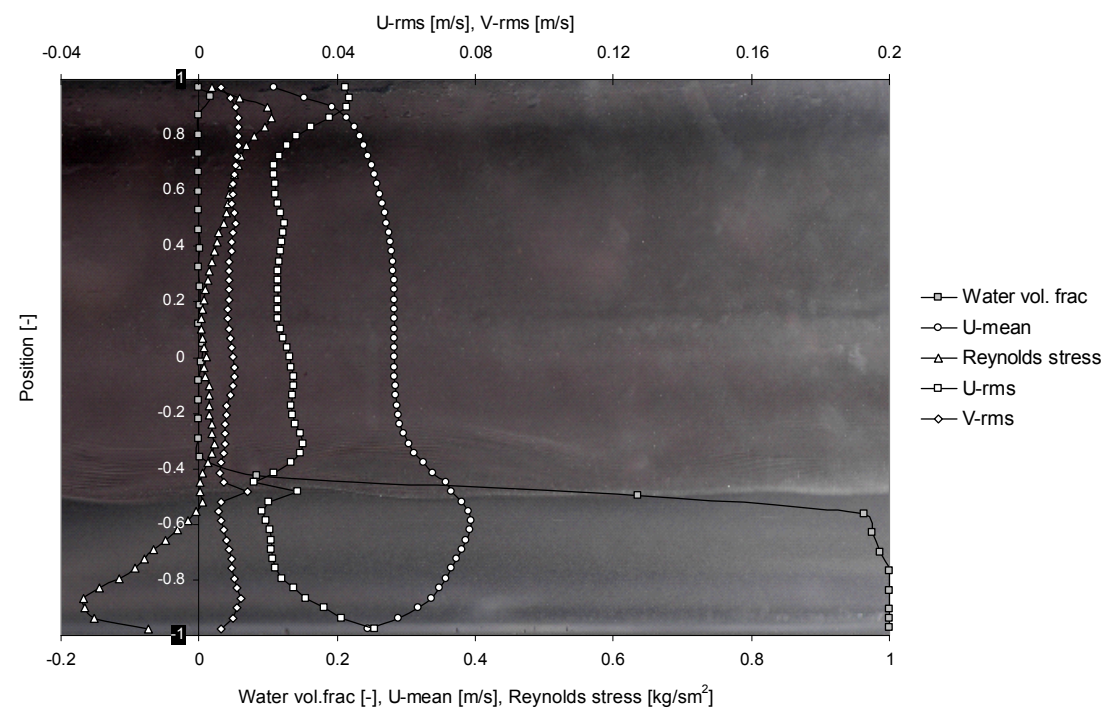

(a)

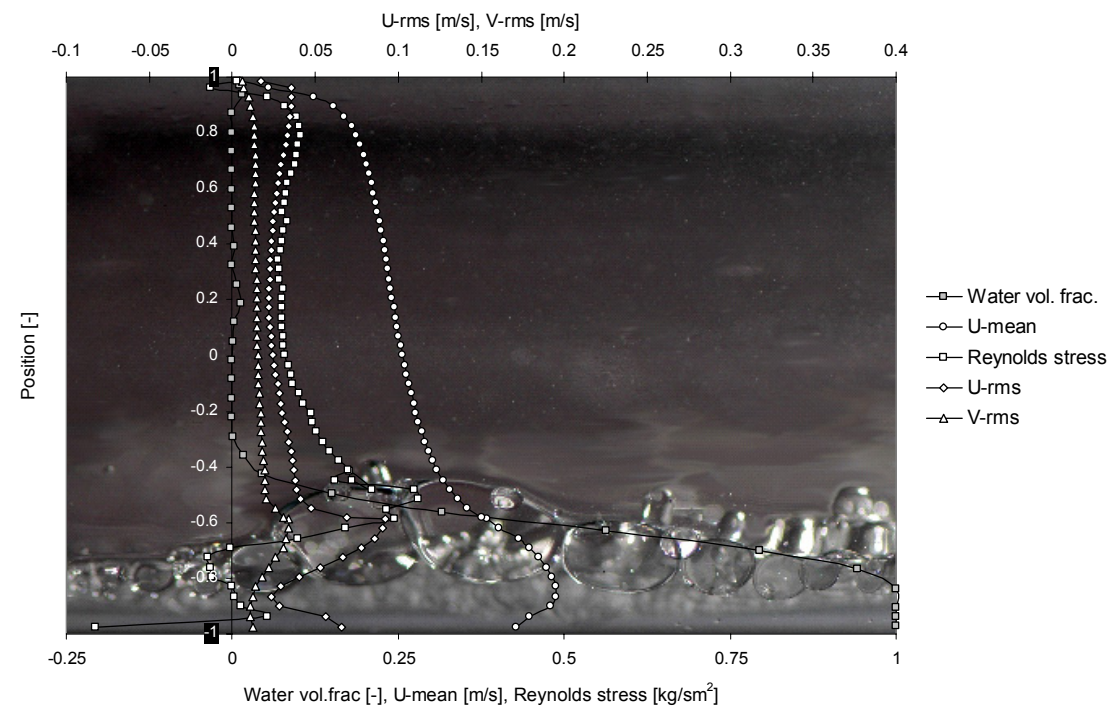

(b)

Figure 7: Mean axial velocity and turbulence measurements for downward flow: (a) $-1^{\circ}$, (b) $-5^{\circ}$.

interface compared to the results at $-1^{\circ}$. This may be due to the slightly wavy structure of the interface. 


\section{Conclusions}

The effect of upward and downward inclination during oil-water pipe flow on flow regimes, water hold-up, slip ratio, velocity and turbulence profiles were investigated experimentally. The experiments were performed in a $15 \mathrm{~m}$ long, 56 mm diameter, inclinable steel pipe using Exxsol D60 oil and water as test fluids. The test pipe inclination was changed in the range from $5^{\circ}$ upward to $5^{\circ}$ downward. All experiments were performed at mixture velocity $0.25 \mathrm{~m} / \mathrm{s}$ and inlet water cut 0.25 . The mean axial velocity and turbulence profiles were measured using PIV, and gamma densitometry is used to measure the cross sectional distribution of oil and water phases. The flow regimes were determined by visual observations. The results were also compared with published inclined oil-water flow studies.

The stratified flow regime was observed with smooth interface for horizontal flow. However, at $+1^{\circ}$ stratified flow with mixing at the interface was observed due to increased slip between oil and water phases. The interface was found to become wavier as the degree of inclination increased from the horizontal. The oil-water interface is noticeably wavier and more irregular at $+5^{\circ}$. Stratified flow with clear interface was observed in downward flow at $-1^{\circ}$. No sign of mixing at the interface is observed in contrast with the results at $+1^{\circ}$. Nevertheless, significant mixing effect at the interface was observed at $-5^{\circ}$ probably because of increased water velocity and tendency of water to disperse the oil. In general, the present observations on flow regimes show a good consensus with previous investigations.

The measured water hold-up in almost all instances are significantly different from the input water volume fraction. As expected, the slip ratio and water holdup increased with pipe inclination, from $-5^{\circ}$ to $+5^{\circ}$. In general, the increases are more prominent in the upward inclinations, while in the downward inclination the increase was more moderate. In addition, the water hold-up and slip ratio are very dependent on the pipe inclination when the pipe is nearly horizontal $\left(-1^{\circ}\right.$ and $+1^{\circ}$ ).

The maximum mean axial velocity is observed in the oil phase for horizontal and upwardly inclined flows whereas it is located in the water phase for downwardly inclined flows. At $+5^{\circ}$, the gravity effects overcome the flowing momentum of the water phase resulting in backflow of thin water layer very close to the bottom pipe wall. In general, higher U-rms, V-rms and Reynolds stress values are observed close to the wall due to large mean axial velocity gradients. A damping effect of Reynolds stresses is observed close to the interface due to stable density stratification for horizontal and near horizontal flows. This may be due to the suppression of turbulence fluctuations normal to the interface by gravitational forces. Surprisingly, larger Reynolds stress values are observed towards the interface at pipe inclination $+5^{\circ}$. In this case, the rolling motion of the phases around the interface generates interfacial waves enhancing turbulence fluctuations close to the oil-water interface. Slightly higher Reynolds stress values are observed close to the interface at pipe inclination $-5^{\circ}$. 


\section{References}

[1] Rodrigues, O.M.H. \& Oliemans, R.V.A., Experimental study on oil-water flow in horizontal and slightly inclined pipes, Int. J. Multiphase Flow, 32, pp. 323-343, 2006.

[2] Lum, J.Y.L., Al-Wahaibi, T. \& Angeli, P., Upward and downward inclination oil-water flows, Int. J. Multiphase Flow, 32, pp. 413-435, 2006.

[3] Hasan, N.M. \& Azzopardi, B.J., Imaging stratifying liquid-liquid flow by capacitance tomograpy, Flow measurement and instrumentation, 18, pp. 241-246, 2007.

[4] Fairuzov, Y.V., Transient gravity-driven countercurrent two-phase liquidliquid flow in horizontal and inclined pipes, Int. J. Multiphase Flow, 29, pp. 1759-1769, 2003.

[5] Scott, G.M., A study of two phase liquid-liquid flow at variable inclinations, M.S. Thesis, University of Texas at Austin, USA, 1985.

[6] Lum, J.Y.L., Lovick, J. \& Angeli, P., Low inclination oil-water flows, Canad. J. Chem. Eng., 82, pp. 303-315, 2004.

[7] Abduvayt, P., Manabe, R., Watanabe, T. \& Arihara, N., Analysis of oilwater flow tests in horizontal, hilly-terrain and vertical pipes, In: Proc. Annual SPE Tech. Conf., Houston, Texas (SPE 90096), in CD ROM, 2004.

[8] Cox, A.L., A study of horizontal and downhill two-phase oil-water flow, M.S. Thesis, University of Texas at Austin, USA, 1985.

[9] Oddie, G., Shi, H., Durlfosky, L.J., Aziz, K., Pfeffer, B. \& Holmes, J.A., Experimental study of two and three phase flow in large diameter inclined pipes, Int. J. Multiphase flow, 29, pp. 527-558, 2003.

[10] Lum, J.Y.L., Lovick, J. \& Angeli, P., Low inclination oil-water flows, Canad. J. Chem. Eng., 82, pp. 303-315, 2004. 